ESAIM: PROCEEDINGS, September 2005, Vol.14, 41-47

Eric Cancès \& Jean-Frédéric Gerbeau, Editors

DOI: $10.1051 /$ proc:2005004

\title{
A MODELING OF BIOSPRAY FOR THE UPPER AIRWAYS*
}

\author{
C. Baranger ${ }^{1}$, L. Boudin ${ }^{2}$, P.-E. Jabin ${ }^{3}$ And S. MAnCini ${ }^{4}$
}

\begin{abstract}
We here deal with a model of therapeutic sprays for the upper airways. We aim to model both inhaled and injected sprays. We propose a numerical solver for the kinetic equation which underlies our model, using a particle method. Eventually, we present two numerical tests for simple geometries of the airways.
\end{abstract}

Résumé. Nous proposons dans ce travail une modélisation du comportement d'un brouillard de gouttelettes à but thérapeutique dans les voies respiratoires supérieures. Notre objectif est de mettre en place un modle pouvant représenter à la fois des sprays inhalés et des sprays injectés. Ce modèle est porté par une équation cinétique, pour laquelle nous présentons une résolution numérique par méthode particulaire. Enfin, nous donnons deux cas-tests pour des conduits respiratoires à géométrie simple.

\section{INTRODUCTION}

Kinetic models are classically used to describe with a statistical point of view a set of numerous small droplets. In many industrial applications, they are coupled with classical fluid models to become the so-called fluid-kinetic models. Those models were first introduced by Williams [Wi] in the context of the combustion theory. O'Rourke [ORo] proposed a modeling of sprays in the nuclear context by a kinetic equation, that took into account many physical phenomena (transport, collision, coalescence, etc.). He also performed a generic numerical study of the equation.

We are here especially interested in modeling sprays in the upper airways of the human respiratory system. Sprays are often used as medical treatments for frequent diseases (flu) and for respiratory diseases (asthma). For each situation, one aims to create a spray of droplets with specific characteristics (velocity, size, temperature, stability with respect to coalescence, breakup, collision, etc.). A droplet is filled with some active products, which are to cure the infected zones, but also with other fluids, whose part is to protect the active product inside the droplet till it reaches the infected zones [Co]. We are interested in two kinds of spray: the inhaled sprays and the injected ones.

Both kinds of sprays have been largely experimentally studied during the past years. We shall refer, for instance, to [GCYSC] and to the references therein. Most of the time, those works consist in measuring a spray distribution in the human throat and comparing the measures to some numerical results obtained through

\footnotetext{
* Supported by IN RIA Project Ré o, UR Rocquencourt, Domaine de Voluceau, B.P. 105, 78153 Le Chesnay CEDEX, France

1 CMLA, CNRS UMR 8536, ENS de Cachan, 61 avenue du Président Wilson, 94235 Cachan CEDEX, France,

Celine.Baranger@cmla.ens-cachan.fr

2 Lab. J.-L. Lions, CNRS UMR 7598, Univ. Paris-V I Pierre et Marie Curie, B.C. 187, 75252 Paris CEDEX 05, France, laurent.boudin@upmc.fr

3 Lab. J.-A. Dieudonné, CNRS UMR 6621, Univ. Nice - Sophia-Antipolis, Parc Valrose, 06108 Nice CEDEX 02, France, jabin@math. unice.fr

${ }^{4}$ MAPMO, CNRS UMR 6628, Univ. Orléans, B.P. 6 759, 45067 Orléans CEDEX 2, France, simona.mancini@univ-orleans.fr
}

(c) EDP Sciences, SMAI 2005 
industrial codes. For example, the Kiva code [K2], [K3v], which was initiated for the nuclear industry, was used by Gemci et al. in [GCYSC]. In a next article [GCC], the same authors proposed a model of biospray similar to ours. But they used Kiva for their numerical experiments, so that their model was not exactly the one they numerically proposed.

With this work, which was performed during the CEMRACS $2004^{1}$, we aim to proceed in a different way: we want to provide a model which is specifically dedicated to biosprays. At this level, the reader must understand that this model has no pretention of originality: we see this work as the first step of a wider program on biospray modeling. We here investigate the kinetic equation which models the spray (see [CP]) without taking into account the ambient fluid equations: that means that the fluid quantities, such as the velocity field, are given in our setting. The coupling with fluid equations should be considered as the next step, in a similar way as in $[\mathrm{Du}]$ or $[\mathrm{BDM}]$. Then we should consider a turbulent fluid flow, since it is the case in the human upper airways.

In this paper, we first present our kinetic model and the phenomena taken into account, then we give a numerical solver of the kinetic equation by a particle method, and we eventually use this solver for two numerical tests with simple geometries of the human throat (a cylinder and a bent pipe).

\section{Biospray MODEL}

As already stated, a spray is here considered as a set of numerous small droplets with a statistical point of view. It is described by the so-called probability density function (PDF), which we here denote by $f$. This function depends on time $t \in \mathbb{R}_{+}$, position $x \in \mathbb{R}^{d}, d=1,2$ or 3 , and velocity $v \in \mathbb{R}^{d}$.

In more intricate models, it may depend on other variables such as the droplet radius or its internal energy. We here choose not to pick the radius as a variable of $f$ as a first approximation. As a matter of fact, both injectors and inhalers are manufactured to deliver droplets that share the same geometrical characteristics. When the droplets are inside the airways, their radius may decrease, but we assume that the decreasing is negligible with respect to the other effects.

We also assume that the droplets remain spherical, with a constant and uniform radius $a$.

Finally, we suppose that energy exchanges are negligible during the whole process. In particular, no thermodynamical effects are taken into account.

The quantity $f(t, x, v) d x d v$ is in fact the number of droplets inside the elementary volume of the phase space $[x ; x+d x] \times[v ; v+d v]$ at time $t$. The PDF satisfies a linear Fokker-Planck equation (see [CP $]$ again)

$$
\partial_{t} f+v \cdot \nabla_{x} f+\lambda \nabla_{v} \cdot((u-v) f)=\nu \Delta_{v} f
$$

The third term in (1) means that the main force which the droplets undergo is the drag force. Note that the gravitation effects are here neglected, as a standard assumption: the droplets weight is small enough compared to the drag force. The fluid is mainly responsible for this drag force: $u(t, x)$ is the velocity field of the ambient fluid, and $\lambda$ is the drag coefficient, which depends on both the fluid and the droplets. The right-hand side term in (1) is the diffusion term, and $\nu$ denotes a diffusion coefficient in the velocity space. Both $\lambda$ and $\nu$ are constant in our model since they depend on dynamics of neither the fluid nor the spray. Their values will be given in the next section.

\section{Numerical SOLVER}

In this section we present the discretization of the kinetic equation (1). Our longterm project is to describe and study the evolution of spray droplets in the respiratory human airways. The real geometries of such airways are very intricate, our discretization must hence be suitable to deal with them. For this reason we choose to approximate (1) by means of a particle method, i.e. we follow each particle along its trajectory.

$1_{\text {see http://smai.emath.fr/cemracs/ }}$ 
The particle method has multiple advantages that justify our choice. The method is quite easy to implement and is independent on the grid chosen to discretize the underlying fluid. It is also rather efficient for high dimensions, when one has to take into account many properties of the droplets, mainly if we do not want to have a very accurate approximation of the initial datum.

We recall that the ambient fluid velocity $u=u(t, x)$ is assumed to be given. This leads us to

$$
\begin{aligned}
x^{n+1} & =x^{n}+v^{n} \Delta t^{n}, \\
v^{n+1} & =v^{n}+\lambda\left(u\left(\Delta t^{n}, x^{n}\right)-v^{n+1}\right) \Delta t^{n}+\sqrt{2 \nu} B^{n} \sqrt{\Delta t^{n}} .
\end{aligned}
$$

In (2)-(3), $\Delta t^{n}, x^{n}$ and $v^{n}$ are respectively the time step, the droplet position and velocity at the $n$-th iteration, $B^{n}$ is a Brownian variable following the Gaussian law $\mathcal{N}(0,1)$, and $\lambda$ and $\nu$ are given by the physics of our model:

$$
\begin{gathered}
\lambda=\frac{9 \mu}{2 a^{2} \varrho}, \\
\nu=\frac{27 k T \mu}{8 a^{5} \varrho^{2} \pi},
\end{gathered}
$$

where $a$ is the droplet radius, $\mu$ its viscosity, $T$ its temperature, $\varrho$ its mass density and $k$ the Boltzmann constant.

Concerning the boundary conditions, we are interested in knowing where the droplets leave the computational domain, that is where the spray impacts the airways. Hence, we just perform a test computing whether or not a given droplet still is inside the domain. The computation for a given droplet is then stopped when it leaves the domain, and its final position is kept in a file. The numerical results we present in the next section actually show the distribution of 10000 droplets injected with a velocity only along the $x$ coordinate (i.e. parallel to the longitudinal axe of our domain), when every single droplet has reached a boundary of the domain.

The fluid velocity is described by its value on a fixed finite volume grid. That ensures the compatibility with the next step of the program we intend to follow: the coupling of the kinetic equation (1) with fluid equations. In (3), the quantity $u\left(\Delta t^{n}, x^{n}\right)$ is computed by finding which mesh the droplet is in, and using the associated value of $u$ on the grid. Note that the time step $\Delta t^{n}$ is computed at each iteration with respect to the maximal velocity of the droplets, so that one droplet can never go across two different cells during the same time step:

$$
\Delta t^{n}=\max _{v^{n}} \frac{|\Delta|}{\left|v^{n}\right|}
$$

where $\Delta=\min \left\{\Delta_{x}, \Delta_{y}, \Delta_{z}\right\}$.

We shall not tackle the question of the scheme convergence in this work Nevertheless, the reader should still keep in mind that the method is quite efficient when the fluid velocity field is smooth enough, which is here the case, during a standard human breathing cycle.

\section{Numerical tests}

We perform computations for two kinds of sprays.

The first one simulates an injected spray. At initial time, one injects droplets with a high velocity $\left(v_{\text {in }}=\right.$ $\left.10.0 \mathrm{~m} . \mathrm{s}^{-1}\right)$ in a domain where the human breath is slow $\left(u_{0}=0.100 \mathrm{~m} . \mathrm{s}^{-1}\right)$.

The second one simulates an inhaled spray. One initially inhales almost motionless droplets with a strong breath, i.e. $v_{\text {in }}=1.00 \times 10^{-3} \mathrm{~m} . \mathrm{s}^{-1}$ and $u_{0}=1.00 \mathrm{~m} \cdot \mathrm{s}^{-1}$.

In both cases, there are $10^{4}$ droplets in the spray, which have the same characteristics: radius $a=1.00 \times$ $10^{-5} \mathrm{~m}$, temperature $T=300 \mathrm{~K}$ (standard), mass density $\varrho=1.00 \times 10^{3} \mathrm{~kg} \cdot \mathrm{m}^{-3}$ (water droplets), viscosity $\mu=16.0 \times 10^{-6}$ I.S. With the value of the Boltzmann constant $\left(k=1.38 \times 10^{-23}\right.$ I.S. $)$, we get

$$
\lambda=720 \text { I.S. }, \quad \sqrt{2 \nu}=1.19 \times 10^{-3} \text { I.S. }
$$


The domain itself has the ambition to be a simplified geometry of the human throat. Hence we assume the longitudinal dimension to be $L=7 \mathrm{~cm}$ and the radial one to be $R=2 \mathrm{~cm}$, which can be seen as average sizes for adult human beings.

\subsection{Cylinder}

We first consider a horizontal cylinder (of longitudinal axis $x$ ) of radius $R$ and length $L$. This geometry is mainly dedicated to the numerical validation of the code.

The air velocity is assumed to have a parabolic profile and equals zero on the wall of the cylinder:

$$
u(t ; r, \theta, x)=\left(1-\frac{r^{2}}{R^{2}}\right) u_{0}, \quad 0 \leq x \leq L, \quad 0 \leq r \leq R, \quad 0 \leq \theta<2 \pi
$$

with (almost) standard notations for axisymmetric geometry.

Droplets are injected at point $(x, y, z)=(0,0,0)$ and evolve in the cylinder following the injection dynamics, i.e. a large initial velocity for the droplets and a small one for the fluid, see fig. 1, or the inhalation dynamics, i.e. a small initial velocity for the droplets and a large fluid velocity, see fig. 2 .

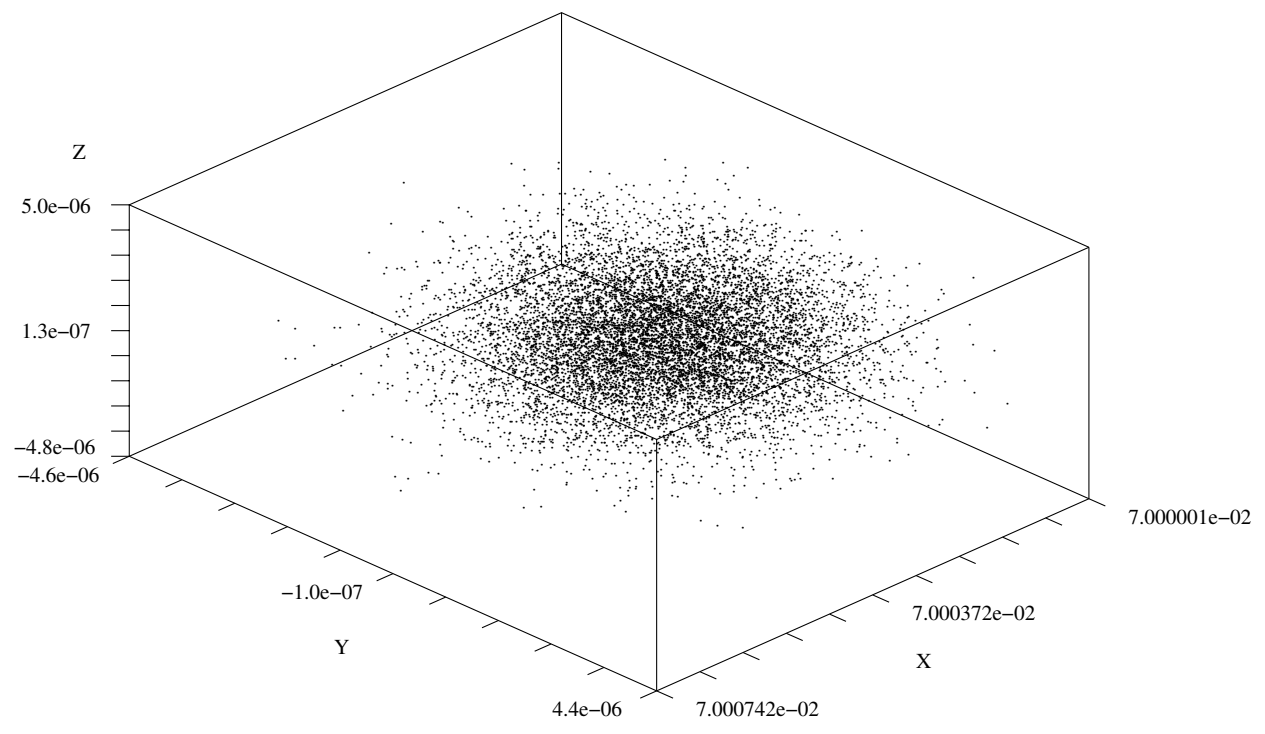

FiguRE 1. Injection for the cylinder (final time).

In both figures, we present the set of droplets at the end of their evolution, i.e. when they reach a border of the cylinder. We notice that in both cases, the droplets reach the opposite side of the cylinder (i.e. $x \geq L$ ) and undergo some diffusion; however, this diffusion is not sufficient to spread the droplets on the borders of the cylinder. The scale in the $x$ coordinate should be precisely equal to $7 \mathrm{~cm}$, but the program only stores the first position after each droplet leaves the computational domain.

Moreover, we may note that the droplets are a little more diffused in the injection test $\left(D_{y}=9.0 \times 10^{-6} \mathrm{~m}\right.$ and $D_{z}=9.8 \times 10^{-6} \mathrm{~m}$, where $D_{y}$ and $D_{z}$ are the width of the region in which droplets arrive along the $y$ and $z$ coordinates), while in the inhalation test, the fluid drags the droplets towards the other side of the cylinder more compactly $\left(D_{y}=3.2 \times 10^{-6} \mathrm{~m}\right.$ and $\left.D_{z}=3.7 \times 10^{-6} \mathrm{~m}\right)$. 


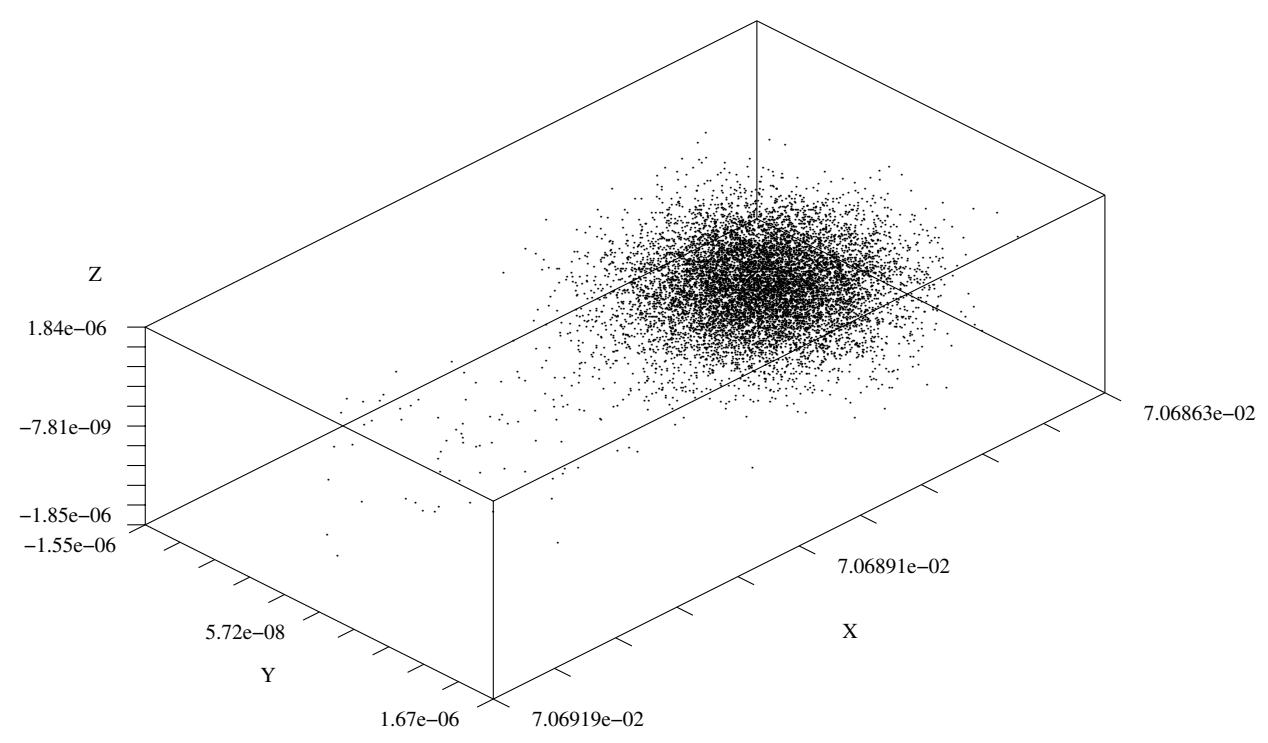

Figure 2. Inhaled spray going out the cylinder (final time).

\subsection{Bent pipe}

The bent pipe is already a more realistic geometry for the human upper airways. The nose and mouth are not separated in this test: the pipe consists in two parallelepipeds of rectangular section (a horizontal one and a vertical one) which are connected (see fig. 3 ).

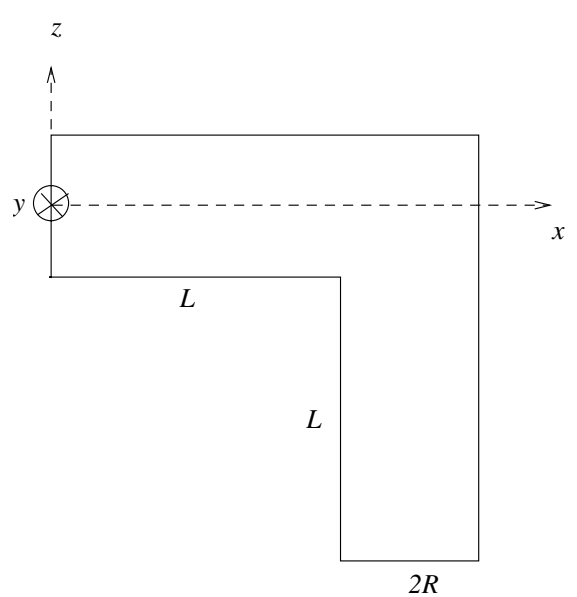

FiguRE 3. Bent pipe geometry. 
The fluid velocity is given by:

$$
u=\left\{\begin{array}{lc}
u_{0}(1,0,0) & 0 \leq x<L, \quad-R \leq y \leq R, \quad-R \leq z \leq R \\
\frac{u_{0}}{\sqrt{2}}(1,0,-1) & L \leq x \leq L+2 R, \quad-R \leq y \leq R, \quad-R<z \leq R \\
u_{0}(0,0,-1) & L \leq x \leq L+2 R, \quad-R \leq y \leq R,-R-L \leq z \leq-R .
\end{array}\right.
$$

We have performed the same two tests than for the cylinder: an injection of droplets and an inhalation. Once again, the droplets have an incoming velocity only parallel to the longitudinal axe $x$ and are injected at point $(x, y, z)=(0,0,0)$. In both cases every particle follows the bent pipe geometry and get to the opposite border, i.e. $x \in[L, L+2 R], y \in[-R, R]$ and $z \leq-R-L$. In fact, even for the injected spray, the droplets go down the pipe and do not stop at the the throat level $(z \simeq 0=$. That means that the drag force is here too strong: the droplets almost immediately keep the air velocity. It seems to be quite unrealistic for the injection case. There are probably some other effects to be taken into account in future works.

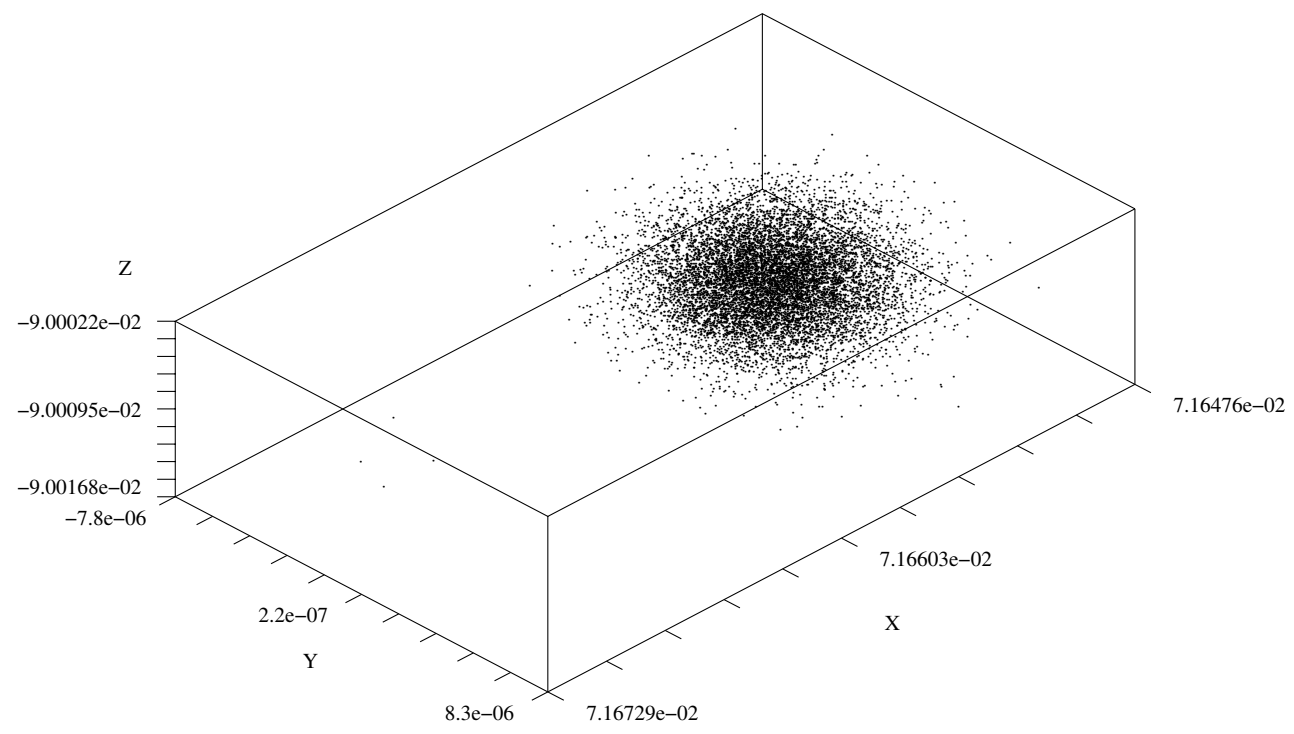

FiguRE 4. Injected spray going out the pipe (final time).

We note that again for the injected spray, see fig. 4, droplets are more diffused $\left(D_{x}=2.53 \times 10^{-5} \mathrm{~m}\right.$ and $D_{y}=1.61 \times 10^{-5} \mathrm{~m}$, where $D_{x}$ and $D_{y}$ are the dimensions of the region where droplets arrive at the end of the bent pipe) when leaving the computational domain, than in the inhalation test $\left(D_{x}=7.28 \times 10^{-6} \mathrm{~m}\right.$ and $D_{y}=5.06 \times 10^{-6} \mathrm{~m}$ ), see fig. 5 . 


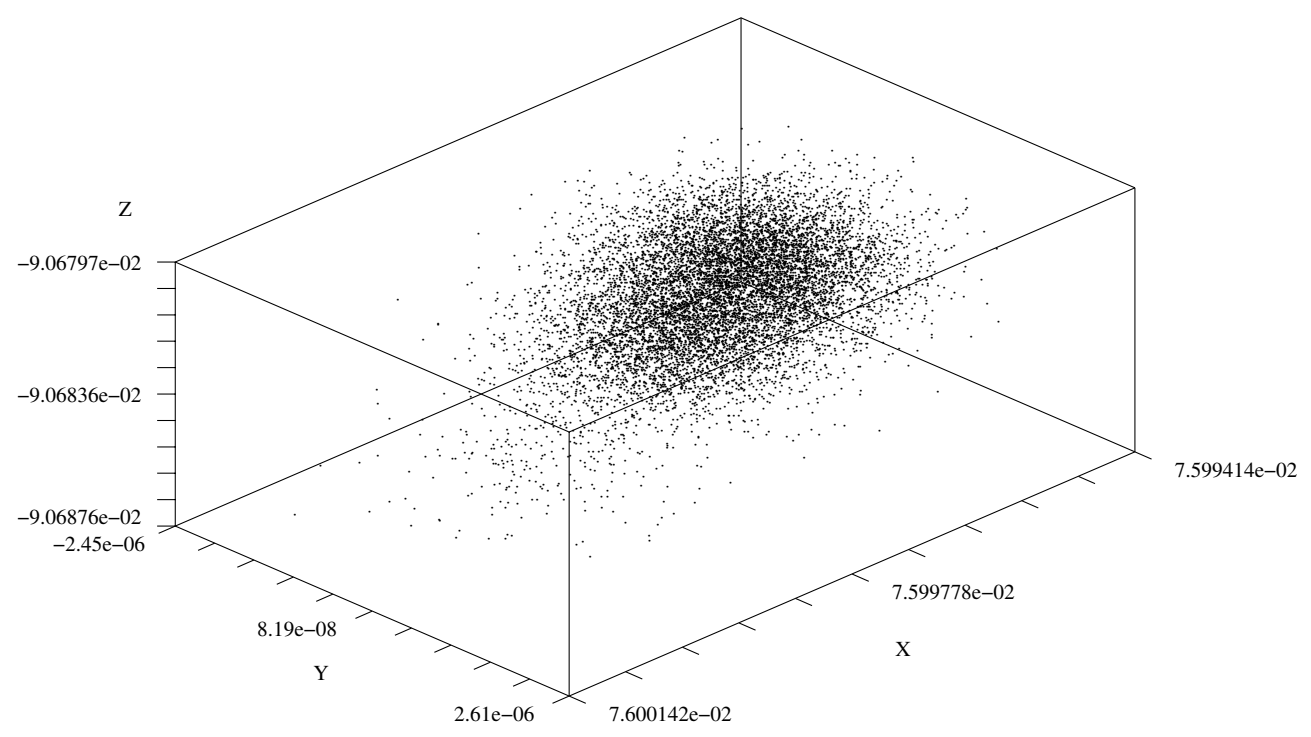

FIGURE 5. Inhaled spray going out the pipe (final time).

\section{REFERENCES}

[K2] A.A. Amsden, P.J. O'Rourke, T.D. Butler. A computer program for chemically reactive flows with sprays. Report \# LA11560-MS, Los Alamos National Laboratory, 1989.

[K3v] A.A. Amsden. Kiva-3V, release 2, improvements to Kiva-3V. Report \# LA-UR-99-915, Los Alamos National Laboratory, 1999.

[BDM] L. Boudin, L. Desvillettes, R. Motte. A modeling of compressible droplets in a fluid. Comm. Math. Sci., 1 (2003), 657-669.

[CP] R. Caflisch, G.C. Papanicolaou. Dynamic theory of suspensions with Brownian effects. SIAM J. Appl. Math., 43 (1983), no. 4, 885-906.

[Co] T.E. Corcoran. Medical nuebulizers: device design and spray deposition with in the human throat. PhD Thesis, Bioengineering, Carnegie Mellon Univ. (2000).

[Du] J.K. Dukowicz. A particle-fluid numerical model for liquid sprays. J. Comp. Phys., 35 (1980), 229-253.

[GCC] T. Gemci, T.E. Corcoran, N. Chigier. A numerical and experimental study of spray dynamics in a simple throat model. Aerosol Sci. Technol., 36 (2002), 18-38.

[GCYSC] T. Gemci, T.E. Corcoran, K. Yakut, B. Shortall, N. Chigier. Spray dynamics and deposition of inhaled medications in the throa t. Proceedings of ILASS-Europe 2001 (Zurich, 2-6 Spetember 2001 ).

[ORo] P.J. O'Rourke. Collective drop effects on vaporizing liquid sprays. PhD Thesis, Los Alamos National Laboratory, 1981.

[Wi] F.A. Williams. Combustion theory, second edition. Benjamin Cummings, 1985. 